\title{
A Solution to the Pre-Main-Sequence Accretion Problem
}

\author{
Paolo Padoan, Alexei Kritsuk and Michael L. Norman \\ Department of Physics and Center for Astrophysics and Space Sciences, University of \\ California, San Diego, \\ 9500 Gilman Drive, La Jolla, CA 92093-0424; ppadoan@ucsd.edu, \\ akritsuk@cosmos.ucsd.edu,mnorman@cosmos.ucsd.edu \\ Åke Nordlund \\ Astronomical Observatory / NBIfAFG, Juliane Maries Vej 30, DK-2100, Copenhagen, \\ Denmark;aake@astro.ku.dk
}

\begin{abstract}
Accretion rates of order $10^{-8} \mathrm{M}_{\odot} \mathrm{yr}^{-1}$ are observed in young pre-mainsequence (PMS) stars of approximately a solar mass with evidence of circumstellar disks. The accretion rate is significantly lower for PMS stars of smaller mass, approximately proportional to the second power of the stellar mass, $\dot{M}_{\text {accr }} \propto M^{2}$. The traditional view is that the observed accretion is the consequence of the angular momentum transport in isolated circumstellar disks, controlled by disk turbulence or self-gravity. However, these processes are not well understood and the observed accretion, a fundamental aspect of star formation, remains an unsolved problem. In this Letter we propose the stellar accretion rate is controlled by accretion from the large scale gas distribution in the parent cloud, not by the isolated disk evolution. Approximating this process as Bondi-Hoyle accretion onto the star-disk system, we obtain accretion rates comparable to the observed ones. We also reproduce the observed dependence of the accretion rate on the stellar mass. These results are based on realistic values of the ambient gas density and velocity, as inferred from numerical simulations of star formation in self-gravitating turbulent clouds.
\end{abstract}

Subject headings: accretion, accretion disks - turbulence - stars: formation, pre-main-sequence - ISM: kinematics and dynamics

\section{Introduction}

Accretion rates of order $10^{-8} \mathrm{M}_{\odot} \mathrm{yr}^{-1}$ are observed in young pre-main-sequence (PMS) stars of mass, $M \sim 1 \mathrm{M}_{\odot}$. Less massive stars and brown dwarfs are also found to accrete, at 
a rate, $\dot{M}_{\text {accr }}$, decreasing with the stellar mass, $M, \dot{M}_{\text {accr }} \propto M^{2}$. A compilation of most accretion rates detected to date is shown in Figure 1. It includes PMS stars and brown dwarfs of $\approx$ 0.5-3 Myr of age (Natta et al. 2004; White \& Hillenbrand 2004; Muzerolle et al. 2004, and references therein). The origin of such accretion rates and their dependence on the stellar mass is not understood. As accreting stars appear to be surrounded by circumstellar disks, it is usually suggested that the accretion is due to the viscous evolution of the disks, controlled by some form of "turbulent viscosity". The angular momentum transport necessary to match the observed accretion rate may result from self-gravity (e.g. Larson 1989; Laughlin \& Bodenheimer 1994), magneto-hydrodynamic turbulence (e.g. Gammie 1996; Stone et al. 2000), or hydrodynamic turbulence (e.g. Klahr \& Bodenheimer 2003). However, circumstellar disks around PMS stars of few Myr of age contain only a few percent of the stellar mass and have a very low fractional ionization. As a consequence, both self-gravity and magnetic coupling may be ineffective, leaving perhaps only baroclinic instability as a viable source of turbulence (see Klahr \& Bodenheimer 2003, and references therein).

Given the uncertainty in the source of disk turbulence and our limited understanding of turbulent transport mechanisms, it is possible to compare the predicted "viscous" disk evolution with the observations only assuming ad hoc values of the viscosity parameter (Hartmann et al. 1998). Furthermore, PMS stars are always found in association with their parent clouds. For example, most PMS stars in Taurus are confined within filaments of dense gas (Hartmann 2002). Therefore, we should expect an interaction of PMS stars and their circumstellar disks with the surrounding gas, and the assumption of an isolated disk evolution may be incorrect.

In this work we find that accretion from the large scale may be important, and may even account for most of the observed accretion rate. For the purpose of discussion, we propose to consider two main phases of PMS evolution: i) The formation phase and ii) the large scale post-formation accretion phase. The formation phase is roughly a spherical accretion. It ends because the initial mass reservoir is limited by the initial conditions (a more or less distinct turbulent fragment). It results in a contracting central object, the PMS star, and a circumstellar disk. As the disk is initially massive, it is very unstable and rapidly accretes most of its mass onto the central object. After less than $1 \mathrm{Myr}$ the circumstellar disk contains only a few percent of the mass of the system, and the PMS star enters the second phase. The second phase is characterized by mass accretion from the large scale gas distribution in the parent cloud. This large scale accretion may account for most of the estimated protostellar accretion rates. It interacts with the circumstellar disk and may be an important source of disk turbulence, enhancing or even controlling the disk accretion.

In this Letter we show that the large scale accretion, approximated as Bondi-Hoyle 
accretion mediated by the circumstellar disk, reproduces the observed accretion rate and its dependence on the stellar mass.

\section{Bondi-Hoyle Approximation for Large Scale Accretion}

We propose a solution to the PMS accretion problem that avoids the necessity of an effective angular momentum transport mechanism in isolated disks. The basic idea is that young accreting PMS stars are not isolated objects. Even if they do not appear to be heavily embedded in protostellar cores, they are still associated with their parent molecular cloud and the accretion from the large scale gas distribution in the cloud cannot be neglected.

The initial formation phase of a star is essentially a spherical accretion. In this phase we assume that self-gravity, shocks, turbulence and magnetic fields in combination are able to effectively transfer angular momentum outwards. Due to this effective angular momentum transport, a central object containing most of the mass is formed, surrounded by a rotationally supported disk of smaller mass. The angular momentum problem may be more serious when the disk contains only a few percent of the protostellar mass and if the ionization fraction is very low. In this case, self-gravity and magnetic fields may not be responsible for the angular momentum transport, and some alternative source of turbulence is required (see Klahr \& Bodenheimer 2003, and references therein). However, even if disk accretion were almost interrupted due to the lack of angular momentum transport in an isolated disk, accretion from the large scale flow of the parent cloud is unavoidable. This large scale accretion may represent the main mass reservoir for the observed PMS accretion. The accreting material from the large scale is likely to be reprocessed through the disk, and may also be responsible for "activating" the disk accretion it fuels, by destabilizing the disk (e.g. generating disk turbulence).

The accretion from the large scale lacks the spherical symmetry of the initial formation

phase, because it occurs far from hydrostatic equilibrium. The gas flow is initially along the dense filaments where the stars are born, and later originates from the general turbulent flow. In order to estimate the accretion rate, we assume this process can be approximated as Bondi-Hoyle accretion. In the idealized Bondi-Hoyle accretion, the star is in supersonic motion with respect to the gas. The stellar gravitation focuses the stream of gas and generates a shock at the back side of the star. In this shock, excess angular momentum with respect to the star cancels, and the gas can fall onto the star from the back side. The Bondi-Hoyle radius of this accretion flow that eventually falls onto the star spans the range 10-1000 AU for a star-to-gas velocity of $1 \mathrm{~km} / \mathrm{s}$ and stellar masses in the range $0.01-1 \mathrm{M}_{\odot}$ respectively. This idealized Bondi-Hoyle flow may thus interact with the circumstellar disk (radius of 
order $100 \mathrm{AU})$, particularly for small stellar masses.

In a more realistic description of a star-forming cloud, the accreting material is not uniform and carries a finite amount of angular momentum, due to the turbulent nature of the ambient medium. Krumholz et al. (2005) have found that angular momentum may reduce the Bondi-Hoyle accretion rate onto the star. However, most material should accrete onto the star-disk system. Numerical simulations have shown that, even in the presence of angular momentum, the accretion rate into a central region of size as small as one percent of the Bondi-Hoyle radius (hence smaller than the circumstellar disk radius) is generally comparable to the Bondi-Hoyle estimate (Ruffert \& Anzer 1995; Ruffert 1997, 1999). Excess angular momentum with respect to the star may cancel in shocks with the disk. Details depend on the orientation of the accretion flow relative to the disk and on the size of the Bondi-Hoyle radius relative to the disk radius. We use the Bondi-Hoyle accretion rate, $\dot{M}_{\text {accr }} \approx \dot{M}_{\mathrm{BH}}$, as an order-of-magnitude estimate of the accretion from the large scale onto the star-disk system, not directly onto the star. The rate is given by

$$
\dot{M}_{\mathrm{BH}}=\frac{4 \pi G^{2} \rho_{\infty}}{\left(c_{\infty}^{2}+v_{\infty}^{2}\right)^{3 / 2}} M^{2}
$$

(Bondi \& Hoyle 1944; Bondi 1952; Edgar 2004, and references therein). Here, $\rho_{\infty}, c_{\infty}$ and $v_{\infty}$ are the gas density, sound speed and gas velocity relative to the star, at a large distance.

Assuming an average gas density $\rho_{\infty}=2.46 m_{\mathrm{H}} 10^{3} \mathrm{~cm}^{-3}\left(m_{\mathrm{H}}\right.$ is the mass of the proton), a velocity of the gas relative to the star $v_{\infty}=1 \mathrm{~km} / \mathrm{s}$, a sound speed $c_{\infty}=0.2 \mathrm{~km} / \mathrm{s}$ and a stellar mass $M=1 \mathrm{M}_{\odot}$, equation (1) yields $\dot{M}_{\text {accr }} \approx 10^{-8} \mathrm{M}_{\odot} \mathrm{yr}^{-1}$, as observed. Furthermore, as $\rho_{\infty}, c_{\infty}$ and $v_{\infty}$ do not depend on the stellar mass, the accretion rate is proportional to the second power of the stellar mass, $\dot{M}_{\text {accr }} \propto M^{2}$, also consistent with the observations. For a brown dwarf of $0.03 \mathrm{M}_{\odot}$, for example, the above physical parameters give $\dot{M}_{\text {accr }} \approx 10^{-11} \mathrm{M}_{\odot} \mathrm{yr}^{-1}$, consistent with inferred accretion rates for young brown dwarfs.

The values adopted above for $\rho_{\infty}, c_{\infty}$ and $v_{\infty}$ characterize molecular cloud filaments and vary independently of the stellar mass. Their variation is thus a natural cause for the scatter in the observed accretion rates visible in Figure 1, on top of the general trend.

On the scale of a few parsecs, jets and outflows from PMS stars are not the primary source of the turbulence that controls the large scale accretion, as indicated by statistical studies of turbulence in star-forming clouds (Ossenkopf \& Mac Low 2002; Heyer \& Brunt 2004). However, on smaller scale, jets and outflows may directly interact with the accretion flow (eg Arce \& Sargent 2004) and may be another source of the scatter in the observed accretion rates. This is an important topic that should be addressed in future work. 


\section{Large Scale Accretion in Turbulent Clouds}

Because stars are formed in dense filaments, and inherit their initial velocity from the gas in those filaments, the initial large scale accretion rate is always high in young PMS stars ( $\lesssim 1 \mathrm{Myr}$ ), and can easily account for the upper envelope of the observational plot in Figure 1. However, at an age of a few Myr, stellar positions and velocities are not expected to be well correlated with the original filaments, and the large scale accretion rate is expected to decrease with time on the average. The vertical scatter seen in Figure 1 is indeed attributed partly to an age dependence of the accretion rate (e.g. Natta et al. 2004).

To estimate the large scale accretion rate, we use a numerical simulation of star formation in self-gravitating turbulent clouds. We employ a 3-D parallel structured adaptive mesh refinement (AMR) code, Enzo ${ }^{1}$, developed at the Laboratory for Computational Astrophysics by Bryan, Norman and collaborators (Bryan \& Norman 1999; O'Shea et al. 2004). In this simulation, mesh refinement is automatically carried out in collapsing regions in order to always resolve the Jeans length (Truelove et al. 1997). We solve the hydrodynamic equations (the case of magnetized clouds will be presented in a separate work), including self-gravity and a large scale random force to drive the turbulence. We also adopt an isothermal equation of state and periodic boundary conditions. The AMR allows us to achieve a very large dynamical range. The computational box has a size of $5 \mathrm{pc}$ and the gravitational collapse is resolved down to the scale of $2 \mathrm{AU}$. The experiment and the code will be described in detail elsewhere.

In this simulation, a very complex density field is generated by the supersonic turbulence. Highly fragmented dense filaments are the natural result of the turbulent fragmentation process. The densest fragments in the filaments are gravitationally unstable and collapse into protostars. The left panel of Figure 2 shows a typical filament found in the simulation, where several protostars are formed. The protostars appear in the filament as very dense cores, because of the limited dynamical range of the image, but are actually resolved as central objects surrounded by protostellar disks at higher refinement levels, as shown in the right panel of Figure 2.

We can use our experiment to derive the gas density and velocity distributions in the turbulent medium and infer the average and standard deviation of the large scale accretion rate based on the Bondi-Hoyle formula. We interpret this as an estimate of the accretion rate for PMS stars of a few Myr of age, assuming i) stellar velocities and positions are not correlated with the gas after a few Myr from their formation, and ii) the parent cloud has

\footnotetext{
${ }^{1}$ See http://cosmos.ucsd.edu/enzo/
} 
not been (completely) dispersed yet.

We compute the value of the coefficient of equation (1), $4 \pi G^{2} \rho_{\infty} /\left(c_{\infty}^{2}+v_{\infty}^{2}\right)^{3 / 2}$, at each mesh position, adopting the local values of gas density, sound speed and flow velocity for $\rho_{\infty}, c_{\infty}$ and $v_{\infty}$ respectively. The temperature is uniform, $T=10 \mathrm{~K}$, and the sound speed is constant. The mean density is $500 \mathrm{~cm}^{-3}$ and the rms flow velocity is approximately $1.1 \mathrm{~km} / \mathrm{s}$, typical of molecular clouds on the scale of 5 pc. It is important to compute directly the coefficient of equation (1), and not the density and velocity separately, because the density and flow velocity in supersonic turbulence are known to be correlated (Padoan et al. 2001). Averaging over the whole computational volume, we find $\dot{M}_{\text {accr }}=10^{-8.6 \pm 0.8}\left(M / 1 \mathrm{M}_{\odot}\right)^{2} \mathrm{M}_{\odot} \mathrm{yr}^{-1}$. As explained above, this should provide an order-of-magnitude estimate for the accretion rate of PMS stars of a few Myr of age, whose velocities and positions are no longer correlated with the gas in dense filaments. Indeed, the $\pm 1-\sigma$ values of this accretion rate covers approximately the lower half of the plot in Figure 1 (dashed lines).

As an illustration of the large scale accretion rate for younger PMS stars $(\lesssim 1 \mathrm{Myr})$ formed in a turbulent cloud, we can compute the same coefficient from the simulation, but averaging only in regions with density above a certain value. This would mimic the fact that stars are initially born inside dense filaments. In principle, increasing values of the minimum density should correspond to lower ages. As an example, the accretion rate is $\dot{M}_{\text {accr }}=10^{-7.6 \pm 0.7}\left(M / 1 \mathrm{M}_{\odot}\right)^{2} \mathrm{M}_{\odot} \mathrm{yr}^{-1}$, limited to densities above $10^{3} \mathrm{~cm}^{-3}$, and $\dot{M}_{\text {accr }}=$ $10^{-6.6 \pm 0.6}\left(M / 1 \mathrm{M}_{\odot}\right)^{2} \mathrm{M}_{\odot} \mathrm{yr}^{-1}$, for densities above $10^{4} \mathrm{~cm}^{-3}$. The solid lines in Figure 1 show the $\pm 1-\sigma$ values for densities above $10^{4} \mathrm{~cm}^{-3}$. These values can explain the upper envelope of the observational plot as due to large scale accretion on very young PMS stars. A selfconsistent calculation of the large scale accretion rate of PMS stars formed in turbulent clouds will be the subject of future work.

\section{Conclusions}

We have shown that accretion from the large scale gas distribution in the parent cloud can explain the observed PMS accretion rates and their dependence on the stellar mass. This process is approximated as Bondi-Hoyle accretion onto the star-disk system in a turbulent molecular cloud and is distinct from the usual disk accretion, which assumes an isolated disk evolution. The large scale accretion provides both the mass reservoir for the observed accretion and the possibility of destabilizing the disk and "activating" its turbulent transport. Regardless of how accretion is mediated on the smallest scales, it may be controlled by the large scale accretion from the ambient medium. 
An important consequence of this new picture is that the disk lifetime could be longer than the ratio of the disk mass and the accretion rate, because the disk is not the only mass reservoir for the accretion; rather it is a sort of buffer between the large scale flow and the final accretion onto the star. PMS accretion continues until the large scale accretion is significantly diminished by an increased stellar velocity relative to the gas or is interrupted by the cloud dispersion. It may also be interrupted if the star ends up in a region of very low gas density.

This large scale accretion may play an important role in the process of planet formation, because of the induced turbulence in disks that may otherwise be stable. Turbulence may be important because it could speed up the growth of grains, which is usually considered essential for the origin of rocky planets, or because it may trigger or modify direct gravitational collapse.

During the October 2004 brown dwarf conference in Volterra, PP enjoyed stimulating discussions on PMS accretion with Francesco Palla, Antonella Natta and Leonardo Testi, which inspired this work. James Muzerolle and Russel White provided the accretionrate data plotted in Figure 1. Comments from the referee and from Lee Hartmann, Mark Krumholz, Russel White, Lynne Hillenbrand, James Muzerolle, Alyssa Goodman and John Scalo have helped improve this Letter. The numerical simulation was done using the IBM Data Star system at the San Diego Supercomputer Center with support from NRAC award MCA098020S.

\section{REFERENCES}

Arce, H. G., \& Sargent, A. I. 2004, ApJ, 612, 342

Bondi, H. 1952, MNRAS, 112, 195

Bondi, H. \& Hoyle, F. 1944, MNRAS, 104, 273

Bryan, G. L. \& Norman, M. L. 1999, in IMA, Vol. 117, Adaptive Mesh Refinement (SAMR) Grid Methods, ed. S. B. Baden, N. P. Chrisochoides, D. Gannon, \& M. L. Norman, New York: Springer, 165

Edgar, R. 2004, New Astronomy Review, 48, 843

Gammie, C. F. 1996, ApJ, 462, 725

Hartmann, L. 2002, ApJ, 578, 914 
Hartmann, L., Calvet, N., Gullbring, E., \& D’Alessio, P. 1998, ApJ, 495, 385

Heyer, M. H., \& Brunt, C. M. 2004, ApJ, 615, L45

Klahr, H. H. \& Bodenheimer, P. 2003, ApJ, 582, 869

Krumholz, M. R., McKee, C. F., \& Klein, R. I. 2005, ApJ (in press), astro

Larson, R. B. 1989, in The Formation and Evolution of Planetary Systems, 31-48

Laughlin, G. \& Bodenheimer, P. 1994, ApJ, 436, 335

Muzerolle, J., Luhman, K. L., Briceno, C., Hartmann, L., \& Calvet, N. 2004, ApJ, submitted

Natta, A., Testi, L., Muzerolle, J., Randich, S., Comerón, F., \& Persi, P. 2004, A\&A, 424, 603

O’Shea, B. W., Bryan, G., Bordner, J., Norman, M. L., Abel, T., Harkness, R., \& Kritsuk, A. 2004, astro-ph/0403044

Ossenkopf, V., \& Mac Low, M.-M. 2002, A\&A, 390, 307

Padoan, P., Juvela, M., Goodman, A. A., \& Nordlund, Å. 2001, ApJ, 553, 227

Ruffert, M. 1997, A\&A, 317, 793

-. 1999, A\&A, 346, 861

Ruffert, M. \& Anzer, U. 1995, A\&A, 295, 108

Stone, J. M., Gammie, C. F., Balbus, S. A., \& Hawley, J. F. 2000, Protostars and Planets IV, 589

Truelove, J. K., Klein, R. I., McKee, C. F., Holliman, J. H., Howell, L. H., \& Greenough, J. A. 1997, ApJ, 489, L179

White, R. J. \& Hillenbrand, L. A. 2004, ApJ, 616, 998 


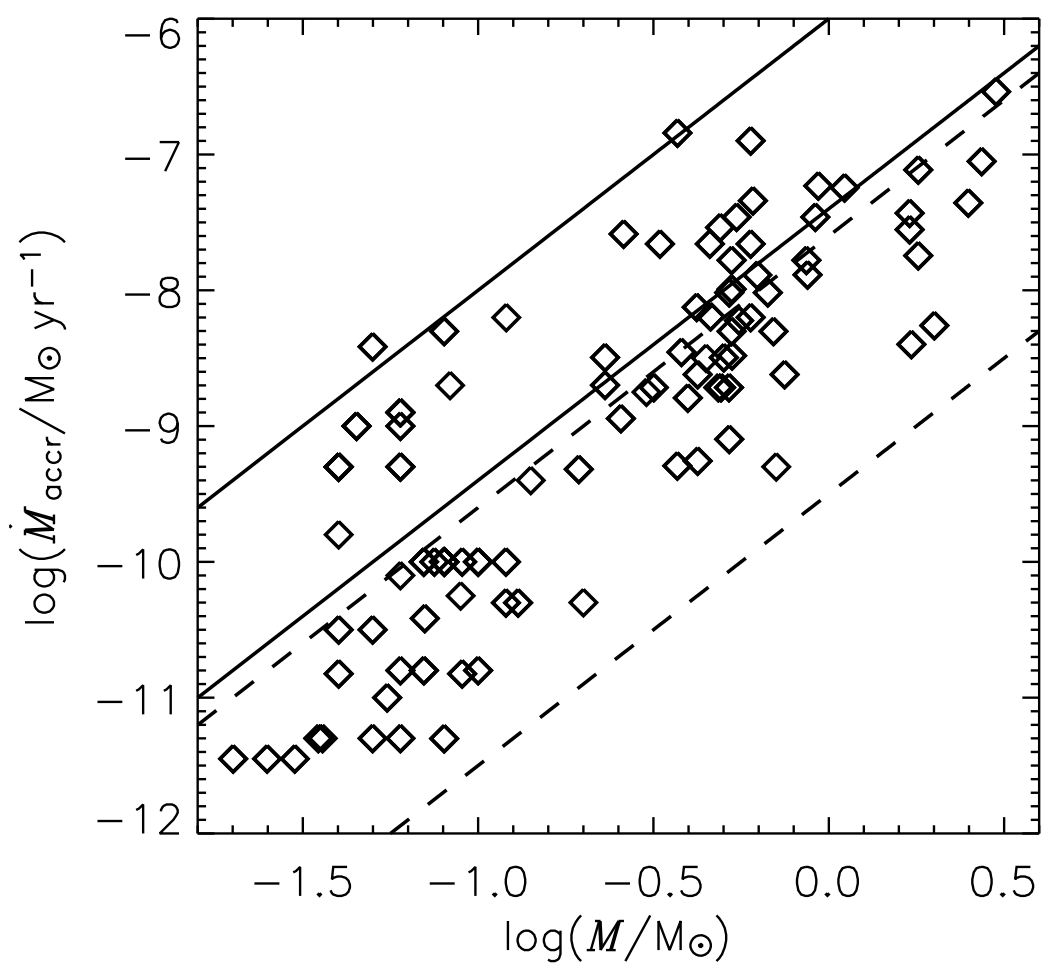

Fig. 1. - Detected accretion rate versus stellar mass including all detections in Natta et al. (2004), White \& Hillenbrand (2004), and Muzerolle et al. (2004) for pre-main-sequence stars and brown dwarfs of $0.5-3 \mathrm{Myr}$ of age. No upper limit has been included in this plot. The two solid lines show the $1-\sigma$ scatter around the $M^{2}$ dependence predicted in $\S 3$ for young PMS stars $(\lesssim 1 \mathrm{Myr})$, assuming a minimum density of $10^{4} \mathrm{~cm}^{-3}$ for the accreting gas. The two dashed lines show the $1-\sigma$ scatter around the $M^{2}$ dependence predicted in $\S 3$ for older PMS stars (few Myr). The lower dashed line roughly corresponds also to the detection limit. 


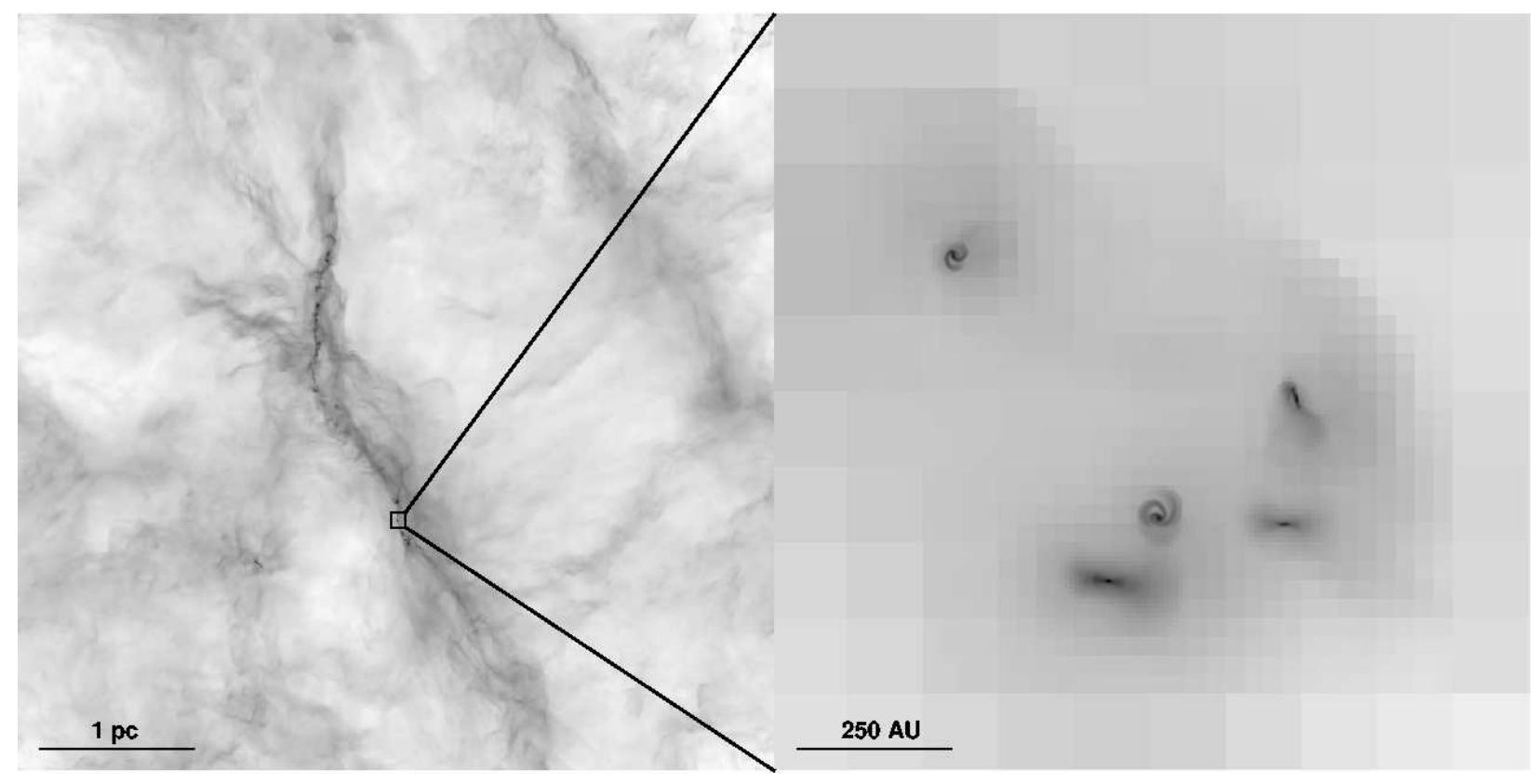

Fig. 2.- Left panel: Logarithm of the projected gas density in our AMR simulation of star formation in a turbulent cloud (see text). Right panel: Higher resolution view of a small region inside the dense filament marked by the small square in the left panel. The small square is drawn larger than its actual size, as the magnification factor between the right and the left panels is 840 . 